\title{
INNOVATIVE INSTRUMENTS IN FORENSIC MEDICINE
}

\author{
Paranitharan $P^{1} \&$ Hulathduwa S.R. ${ }^{2}$ \\ ${ }^{1}$ Faculty of Medicine, University of Kelaniya, Sri Lanka \\ ${ }^{2}$ Facultyof Medical Sciences, University of Sri Jayawardenepura, Sri Lanka
}

\begin{abstract}
Innovation is a hallmark of advancement of science. Forensic professionals, together with other experts and equipment manufacturers, can help to design modern and novel equipment to enhance the quality of work and scientific validity. The major branches, such as Clinical Forensic Medicine and Forensic Pathology, need modern equipment as in other disciplines in Medicine. Creative ideas need support and link to the right individuals.
\end{abstract}

Key words: innovation, sexual assault forensic examination spectacles, forensic portable scanner, nanotechnology

Corresponding author: paran28@gmail.com

\section{INTRODUCTION}

Innovation is a hallmark of advancement in science. Developing countries may face limited resources in both materials and manpower. However, that should not prevent advanced thinking related to a particular field of interest. Ideas carry an extra source of power and the key foundation for greater projects.

Advancing the field of Forensic Medicine from any corner of the world could be done quite effectively due to the technological improvements in connectivity. Considering the need to take greater strides, intellectuals need to continuously work on innovative instruments effectively.

\section{Conceptualization}

Clinical Forensic Medicine and Forensic Pathology are the major fields in Forensic Medicine and the academia needs continuous flow of new information to feel that the field is still vibrant. The conceptualization of an instrument at times may look primitive, but it does pave a path for new ideas. The authors wish to present two innovative instruments, which they believe are useful if made available in day-to-day practice.
A. Sexual Assault Forensic
Examination Spectacles
B. Forensic Portable Scanner

\section{Sexual Assault Forensic Examination Spectacles}

Clinical Forensic Medicine involves the examination of victims following sexual abuse. The interpretation of genital injuries, and proper recording for future reference and audit purposes are vital. Towards this line of thinking, an innovative instrument was designed with the existing ideas. This equipment will not be costly and is easily operable. It can be transported to selected places. The raw materials could also be easily accumulated. 


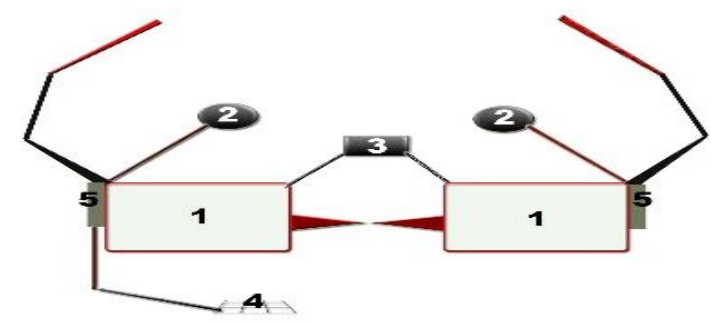

1- Normal Glasses

2- Adjustable magnifying glass

3- Adjustable camera lens with light source

4- Foot pad and switch with adjustable antenna

5- External Memory Device

To do a proper genital examination both hands need to be free and mobile. Therefore, it is prudent that any instrument may be operated by using the foot to adjust the camera, light source and magnifying lens. The clicked images will go to the external memory device in the spectacles and can be later transferred to a permanent storage device. The technology and expertise are already available but it is a matter of diverting the same to be effectively used in Forensic Medicine.

\section{Forensic Portable Scanner}

In developed countries, CT scanners are used as an alternative or together with routine autopsies to identify the cause of death and for various other medico-legal issues. There are autopsies, such as homicides following firearm injuries; deaths following explosions; death of a body packer and in the living; and those smuggling valuables inside body orifices (the so called 'mules'), in which the forensic portable scanner may become valuable to trace foreign material. The proposed scanner is portable; battery operated and contains functions to identify objects and substances of different density. It contains a screen for on the spot visualization and inbuilt memory device for long-term storage.
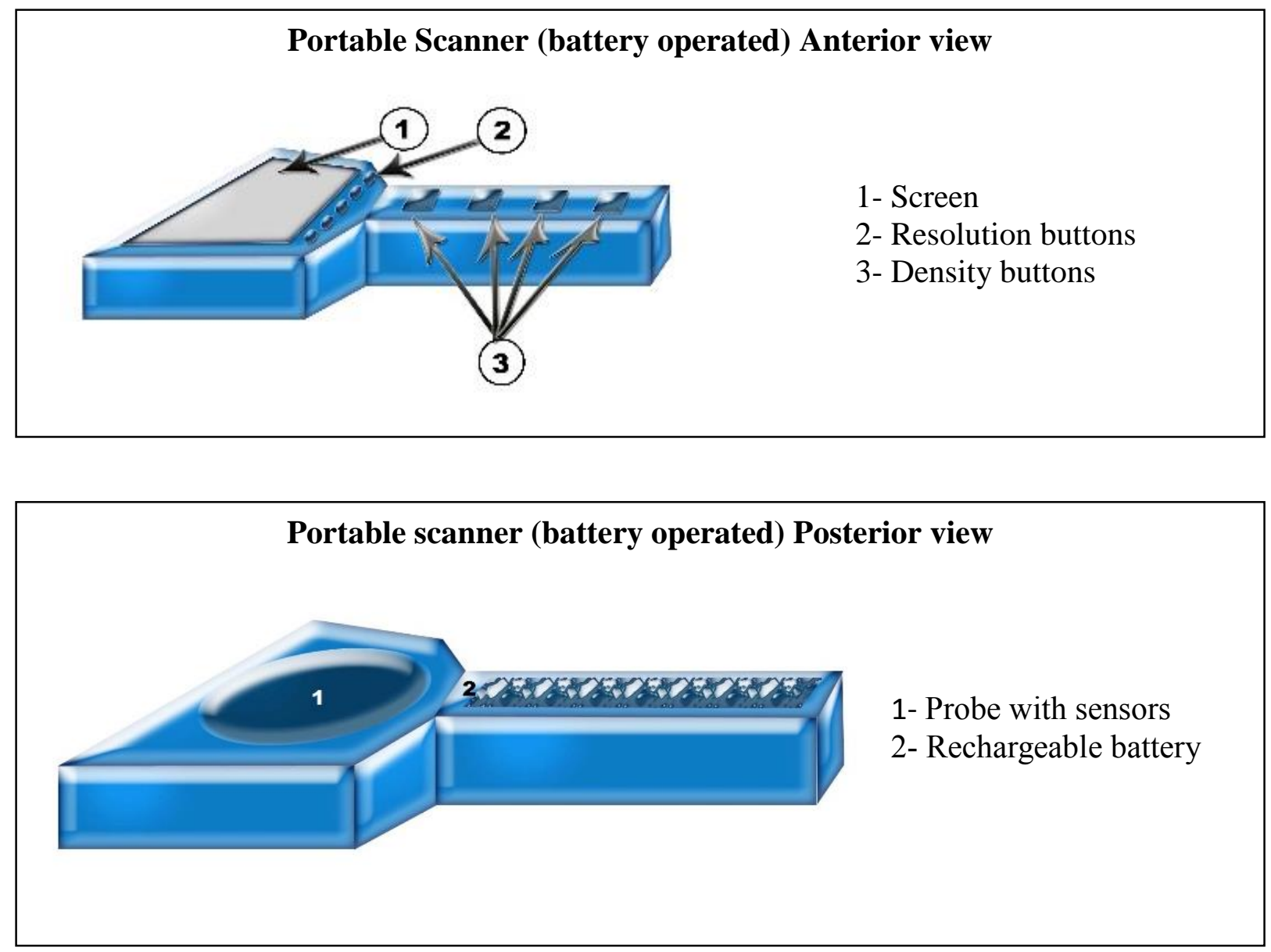
By using the probe on the body surface, the foreign objects or any other suspected materials could be visualized and images could be captured. This can be used even during dissection to trace foreign materials, such as bullets, pellets, detonators following explosions, shrapnel and detached tip of a knife embedded inside a bone and etc. Modified nano-technology could be utilized to transfer waves to body surface and to retrieve them as images. This equipment would be an asset to Forensic Medicine if developed containing all the necessary components.

\section{CONCLUSION}

Modern and innovative instruments in Forensic Medicine will enhance the quality of work and the reliability of scientific findings. The forensic work will reach different levels throughout the world. The collaborative work between different specialists and equipment manufactures would soon become the order of the day.

\section{ACKNOWLEDGEMENT}

The authors wish to acknowledge Dr.PN. Managoda for her assistance in creating the necessary images for this manuscript.

\section{DECLARATION}

The abstract was accepted for oral presentation at the INPALMS held in Bali, Indonesia. 2016

\section{LIMITATIONS}

The authors wish to state that the suggested equipment are merely conceptualized ideas done in the best interest to promote the forensic field. The technology and even the devices may be already available for different purposes.

\section{REFERENCE}

1. The virtopsy approach - 3D optical and radiological scanning and reconstruction in Forensic Medicine. Edited by Michael J. Thali, Peter Vock. 2009. CRC Press.

2. http://www3.gehealthcare.com.au, last accessed on 8.3.2017

3. http://mius.org.uk/portable-ultrasoundscanners/, last accessed on 8.3.2017

4. http://www.theverge.com, last accessed on 8.3.2017

5. https://www.nlm.nih.gov/visibleproofs/exhi bition/newscience.html, last accessed on 8.3.2017 